\title{
Oviposition of the mosquito Aedes aegypti in forest and domestic habitats in Africa
}

\author{
Siyang Xia ${ }^{1}$, Hany Dweck ${ }^{1}$, Joel Lutomiah ${ }^{2}$, Rosemary Sang ${ }^{2}$, Carolyn McBride ${ }^{3}$, Noah \\ Rose $^{3}$, Diego Ayala ${ }^{4}$, and Jeffrey Powell ${ }^{1}$ \\ ${ }^{1}$ Yale University \\ ${ }^{2}$ Kenya Medical Research Institute \\ ${ }^{3}$ Princeton University \\ ${ }^{4}$ Institut de recherche pour le developpement France-Sud
}

July 7, 2020

\begin{abstract}
The theory of ecological divergence provides a useful framework to understand the adaptation of many species to anthropogenic ('domestic') habitats. The mosquito Aedes aegypti, a global vector of several arboviral diseases, presents an excellent study system. Ae. aegypti originated in African forests, but the populations that invaded other continents have specialized in domestic habitats. In its African native range, the species can be found in both forest and domestic habitats like villages. A crucial behavioral change between mosquitoes living in different habitats is their oviposition choices. Forest Ae. aegypti lay eggs in natural water containers like tree holes, while their domestic counterparts heavily rely on artificial containers such as plastic buckets. These habitat-specific containers likely have different environmental conditions, which could drive the incipient divergent evolution of oviposition in African Ae. aegypti. To examine this hypothesis, we conducted field research in two African locations, La Lopé, Gabon and Rabai, Kenya, where Ae. aegypti live in both forests and nearby villages. We first characterized a series of environmental conditions of natural oviposition sites, including physical characteristics, microbial density, bacterial composition, and volatile profiles. Our data showed that in both locations, environmental conditions of oviposition sites did differ between habitats. To examine potential behavioral divergence, we then conducted field and laboratory oviposition choice experiments to compare the oviposition preference of forest and village mosquitoes. The field experiment suggested that forest mosquitoes readily accepted artificial containers. In laboratory oviposition assays, forest and village mosquito colonies did not show a differential preference towards several conditions that featured forest versus village oviposition sites. Collectively, there is little evidence from our study that environmental differences lead to strong and easily measurable divergence in oviposition behavior between Ae. aegypti that occupy nearby forest and domestic habitats within Africa, despite clear divergence between African and non-African Ae. aegypti.
\end{abstract}

\section{Hosted file}

Manuscript.docx available at https://authorea.com/users/340402/articles/467743-ovipositionof-the-mosquito-aedes-aegypti-in-forest-and-domestic-habitats-in-africa 
(a)

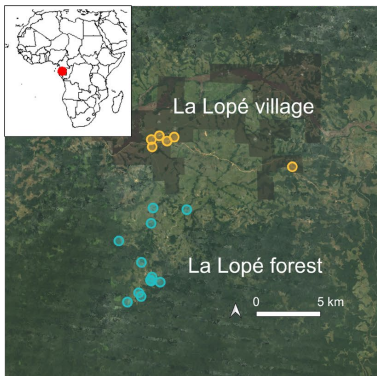

- Forest (b)

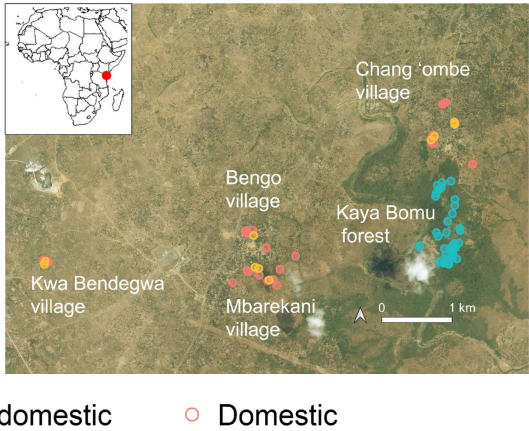

(a)

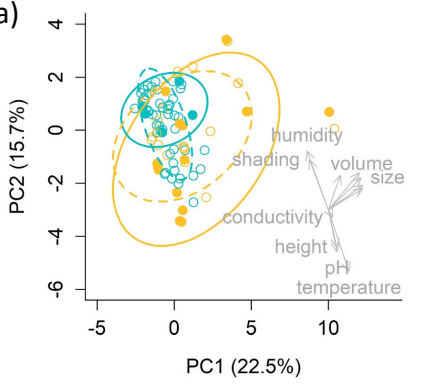

(a)

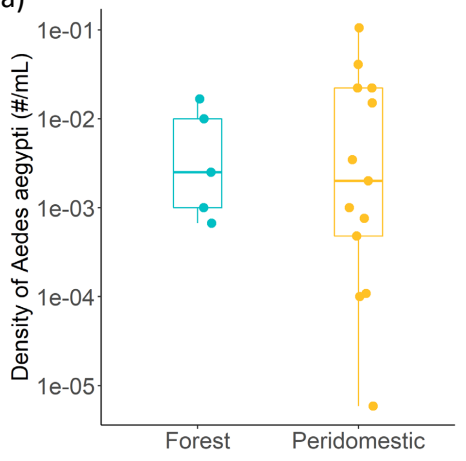

(b)

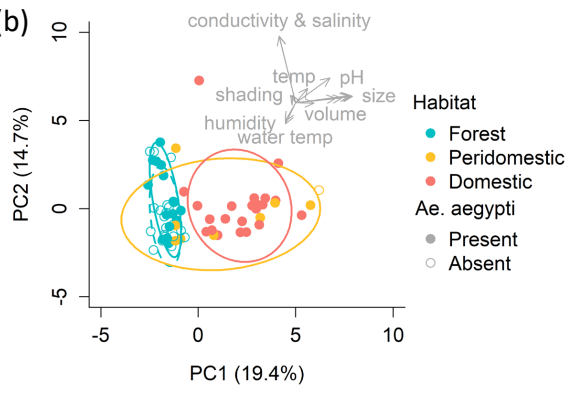

(b)

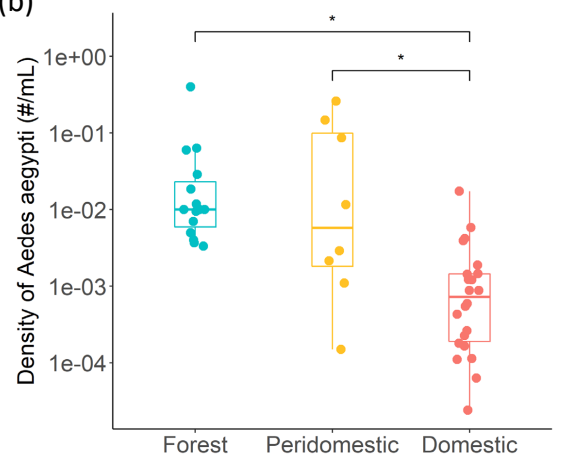


(a)

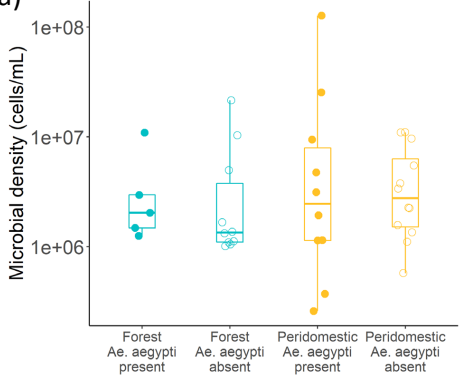

(b)

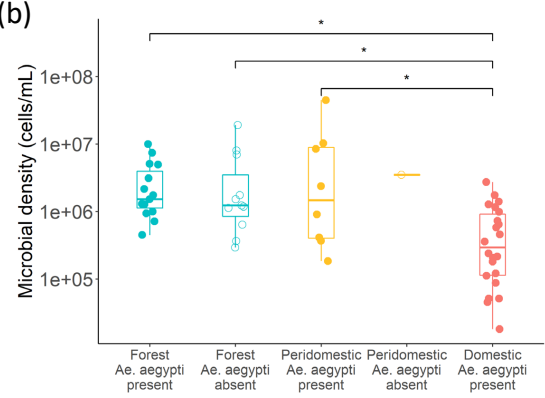

(a)

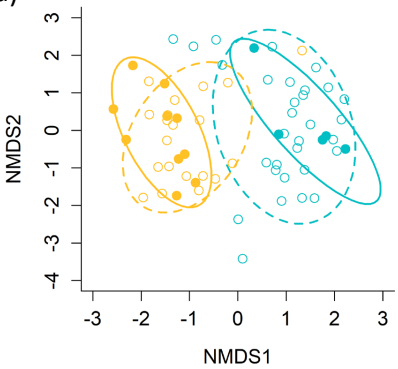

(b)

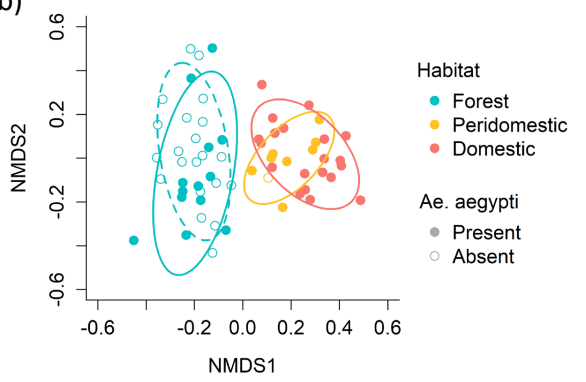




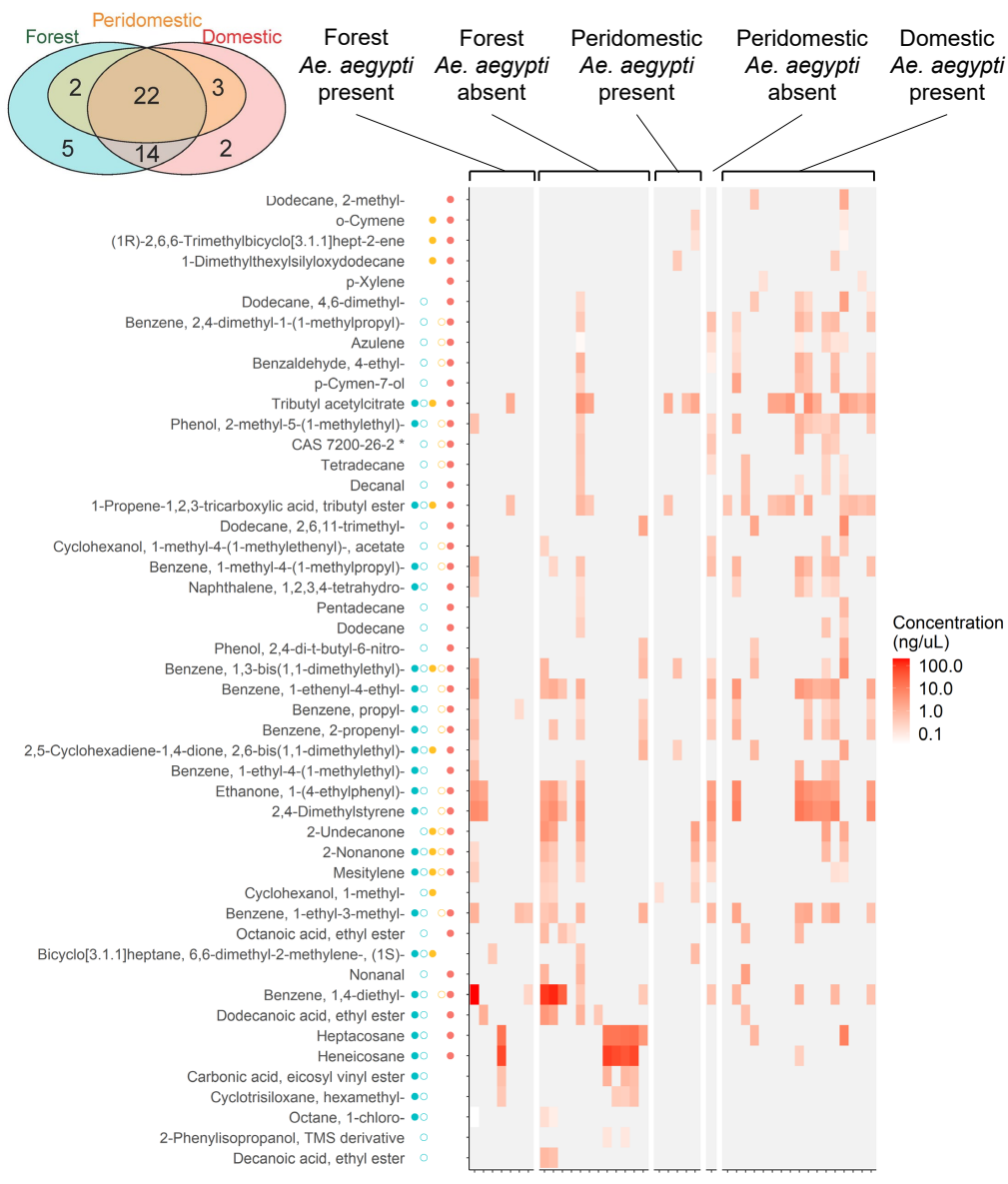


(a)

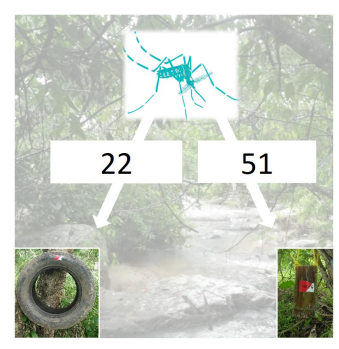

(b)

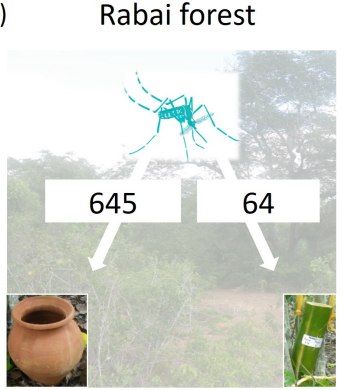

La Lopé village

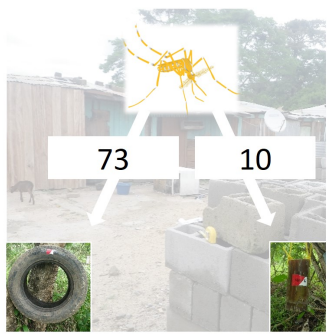

Rabai Bengo village

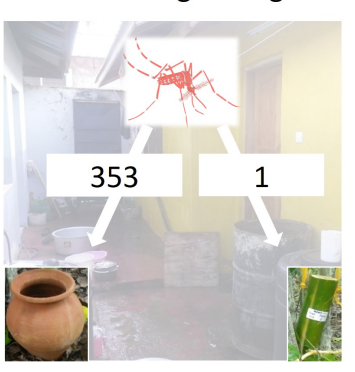

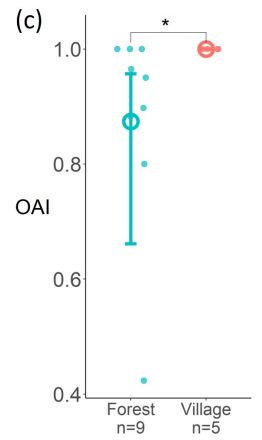
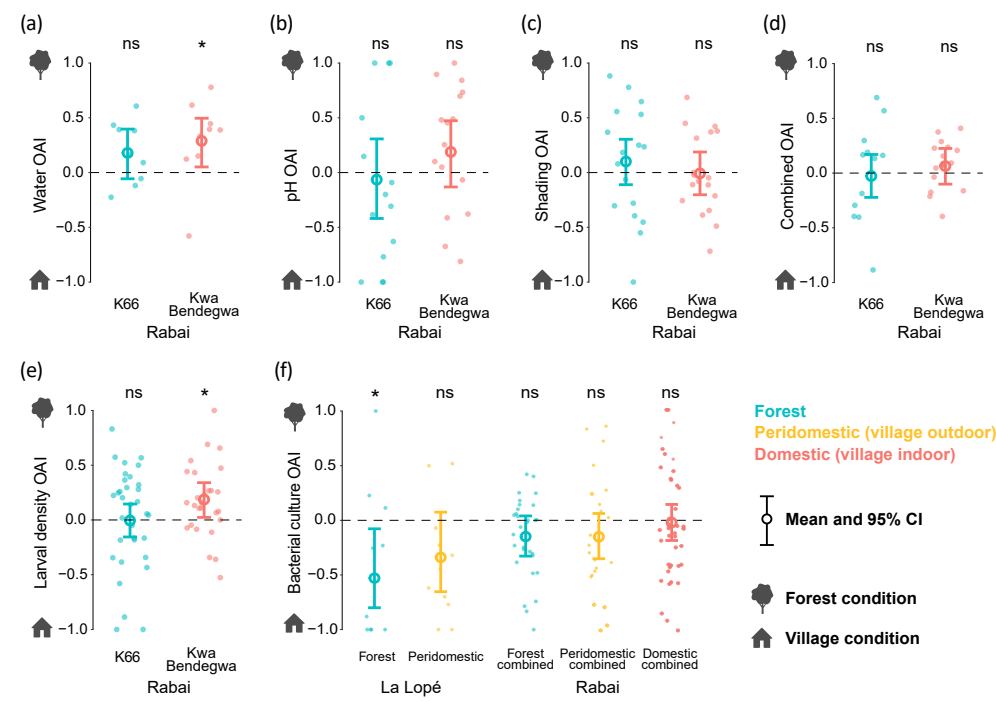

Forest

Peridomestic (village outdoor)

Domestic (village indoor)

T Mean and $95 \% \mathrm{Cl}$

Forest condition

1 Village condition 


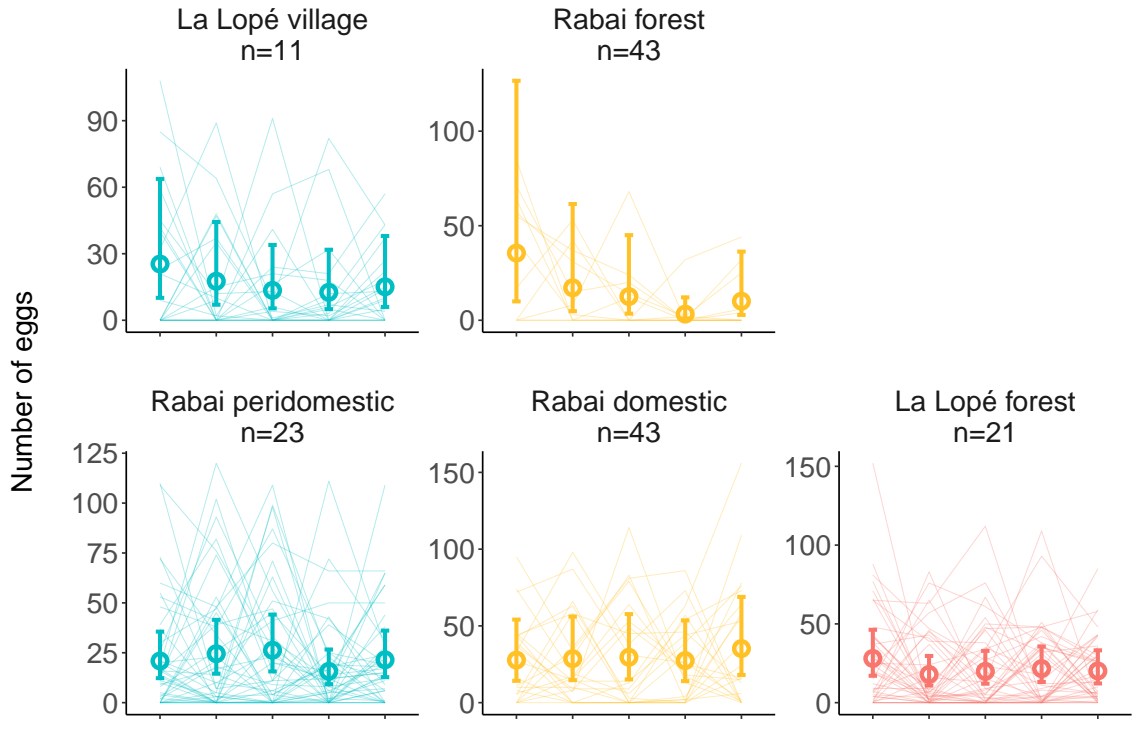

Bacterial density (low to high) 